In order that the general twisted cubic (4) be algebraically rectifiable, it is sufficient, but not necessary, that it be a helix. By demanding algebraic rectifiability of a particular type, we may obtain a condition in terms of the curvature and torsion of (4), which is both necessary and sufficient:

The general twisted cubic (4) may be represented by equations of the form (4), where $t$ is its arc, when and only when it is of constant curvature and torsion.

For a necessary and sufficient condition that (4) may be so represented is that $\left(x^{\prime} \mid x^{\prime}\right)$ is a constant, not zero, and this in turn can be shown to be necessary and sufficient that (4) is a curve of constant curvature and torsion.

WeLIESLEY Colleger,

November 15, 1919.

\title{
NOTE ON LINEAR DIFFERENTIAL EQUATIONS OF THE FOURTH ORDER WHOSE SOLUTIONS \\ SATISFY A HOMOGENEOUS QUADRATIC IDENTITY.
}

BY DR. C. N. REYNOLDS, JR.

(Read before the American Mathematical Society December 31, 1919.)

IN this paper I shall prove that if a given homogeneous linear differential equation of the fourth order has four linearly independent real solutions which satisfy a non-singular homogeneous quadratic identity, then it may be reduced to a form which may be said to be self-adjoint with respect to the third row of the wronskian of any four linearly independent solutions. I shall then evaluate the signature of the quadratic identity in terms of the coefficients of the reduced equation and derive several theorems concerning the zeros of solutions of such equations.

Brioschi* has shown that if $y_{1}(x), y_{2}(x), y_{3}(x)$ and $y_{4}(x)$ are four linearly independent real solutions of the equation

$$
\begin{aligned}
p_{0}(x) y^{\mathrm{Iv}}(x) & +4 p_{1}(x) y^{\prime \prime \prime}(x)+6 p_{2}(x) y^{\prime \prime}(x) \\
& +4 p_{3}(x) y^{\prime}(x)+p_{4}(x) y(x)=0 \quad\left(p_{0}(x) \neq 0\right),
\end{aligned}
$$

* "Les invariants des équations différentielles linéaires," Acta Mathematica (1890), vol. 14, p. 233. 
with real coefficients which together with a sufficient number of their derivatives are continuous, and if

$$
\sum_{i=1}^{4} \sum_{j=1}^{4} A_{i j} y_{i}(x) y_{j}(x) \equiv 0 \quad\left(A_{i j}=A_{j i}\right),
$$

where not all of the $A$ 's are zero; then, by means of a suitable change of variables we can reduce our equations (1) and (2) to the form

where

$$
\begin{aligned}
& v^{\mathrm{IV}}(z)+6 q_{2}(z) v^{\prime \prime}(z)+9 q_{2}{ }^{\prime}(z) v^{\prime}(z) \\
& +\left[3 q_{2}^{\prime \prime}(z)+C\right] v(z)=0 \quad\left(z=f(x), \quad f^{\prime}(x) \neq 0\right), \\
& \sum_{i=1}^{4} \sum_{j=1}^{4} A_{i j} v_{i}(z) v_{j}(z) \equiv 0 \quad\left(A_{i j}=A_{j i}\right),
\end{aligned}
$$$$
\Delta \equiv\left|\begin{array}{llll}
v_{1}(z), & v_{2}(z), & v_{3}(z), & v_{4}(z) \\
v_{1}{ }^{\prime}(z), & v_{2}^{\prime}(z), & v_{3}{ }^{\prime}(z), & v_{4}{ }^{\prime}(z) \\
v_{1}{ }^{\prime \prime}(z), & v_{2}^{\prime \prime}(z), & v_{3}{ }^{\prime \prime}(z), & v_{4}{ }^{\prime \prime}(z) \\
v_{1}^{\prime \prime \prime}(z), & v_{2}^{\prime \prime \prime}(z), & v_{3}{ }^{\prime \prime \prime}(z), & v_{4}{ }^{\prime \prime \prime}(z)
\end{array}\right| \equiv\left(v_{1}, v_{2}{ }^{\prime}, v_{3}{ }^{\prime \prime}, v_{4}{ }^{\prime \prime \prime}\right)
$$

is a constant different from zero. Cels* has called the equation satisfied by the cofactors of $v_{i}^{\prime \prime}(z)(i=1,2,3,4)$ in $\Delta$ the "equation adjoint to (3) with respect to the third row of $\Delta . "$ By repeated differentiation of $\left(v_{1}, v_{2}{ }^{\prime}, v_{3}{ }^{\prime \prime \prime}\right)$ we find that it satisfies (3). This equation may therefore be said to be self-adjoint with respect to the third row of $\Delta$.

If we now define $v_{i}(z)$ by means of the boundary conditions

$$
\begin{aligned}
v_{i}{ }^{(i-1)}(\zeta) & =1, \quad(i, k=1,2,3,4 ; \\
v_{i}{ }^{(k-1)}(\zeta) & =0, \quad k \neq i)
\end{aligned}
$$

then $v(z)$, any solution of (3), may be expressed in the form

$$
v(z)=v(\zeta) v_{1}(z)+v^{\prime}(\zeta) v_{2}(z)+v^{\prime \prime}(\zeta) v_{3}(z)+v^{\prime \prime \prime}(\zeta) v_{4}(z)
$$

In particular, we can so express the cofactors of $v_{i}{ }^{\prime \prime}(z)$ in $\Delta$ :

$$
\begin{array}{rlrl}
\left(v_{2}, v_{3}{ }^{\prime}, v_{4}{ }^{\prime \prime \prime}\right) & = & v_{3}, & \\
-\left(v_{1}, v_{3}{ }^{\prime}, v_{4}{ }^{\prime \prime \prime}\right) & = & -v_{2} & +6 q_{2}(\zeta) v_{4}, \\
\left(v_{1}, v_{2}{ }^{\prime}, v_{4}{ }^{\prime \prime \prime}\right) & =v_{1} & -6 q_{2}(\zeta) v_{3} & -3 q_{2}{ }^{\prime}(\zeta) v_{4}, \\
-\left(v_{1}, v_{2}{ }^{\prime}, v_{3}{ }^{\prime \prime \prime}\right) & = & 6 q_{2}(\zeta) v_{2}-3 q_{2}{ }^{\prime}(\zeta) v_{3}-\left[36 q_{2}{ }^{2}(\zeta)-C\right] v_{4} .
\end{array}
$$

* "Sur les équations différentielles linéaires ordinaires," Annales Scient. de l'Ecole Normale Supérieure (1891), vol. 8, 3d ser., p. 341. 
Substituting these values of our three rowed determinants in the expansion of the determinant

$$
\left(v_{1}, v_{2}{ }^{\prime}, v_{3}, v_{4}{ }^{\prime \prime \prime}\right) \equiv 0
$$

in terms of the elements of its third row, we have

$$
\begin{aligned}
& {\left[v_{1}+\left(-3 q_{2}(\zeta)+\frac{1}{2}\right) v_{3}-3 q_{2}{ }^{\prime}(\zeta) v_{4}\right]^{2}} \\
& -\left[v_{1}+\left(-3 q_{2}(\zeta)-\frac{1}{2}\right) v_{3}-3 q_{2}{ }^{\prime}(\zeta) v_{4}\right]^{2} \\
& -\left[v_{2}-6 q_{2}(\zeta) v_{4}\right]^{2}+C v_{4}{ }^{2} \equiv 0
\end{aligned}
$$

Therefore, the signature of the quadratic form in (4) is zero if $C$ is positive, two if $C$ is negative.

If $v_{1}(z)$ and $v_{2}(z)$ are any solutions of (3) then

$S\left(v_{1}, v_{2}\right) \equiv\left(v_{1}^{\prime \prime \prime}+6 q_{2} v_{1}{ }^{\prime}+3 q_{2}{ }^{\prime} v_{1}\right)\left(v_{2}{ }^{\prime \prime \prime}+6 q_{2} v_{2}{ }^{\prime}+3 q_{2}{ }^{\prime} v_{2}\right)$

is constant since

$$
+C\left(v_{1}{ }^{\prime \prime} v_{2}-v_{1}{ }^{\prime} v_{2}{ }^{\prime}+v_{1} v_{2}{ }^{\prime \prime}+6 q_{2} v_{1} v_{2}\right)
$$

$$
\frac{d}{d z} S\left(v_{1}, v_{2}\right) \equiv 0 \text {. }
$$

If $v(\zeta)=v^{\prime}(\zeta)=0$ then $\dot{S}(v, v)=v^{\prime \prime \prime}{ }^{2}(\zeta) \geqq 0$. Therefore, no solution $v(z)$ of $(3)$ for which $S(v, v)<0$ can have a multiple zero. Similarly we find that

If $C<0$ and $S(v, v)<0$ then $v(z)$ has no zeros.

If $C\left[S\left(v_{1}, v_{1}\right) S\left(v_{2}, v_{2}\right)-S^{2}\left(v_{1}, v_{2}\right)\right]>0$ then $v_{1}(z)$ and $v_{2}(z)$ have no zeros in common.

Brioschi has shown that if $C>0$ then any solution of (3) is a bilinear form in the solutions of

$$
\begin{gathered}
u^{\prime \prime}(z)+\left[\frac{3}{2} q_{2}(z)+r\right] u(z)=0, \quad 4 r^{2}=C, \quad r>0, \\
w^{\prime \prime}(z)+\left[\frac{3}{2} q_{2}(z)-r\right] w(z)=0 .
\end{gathered}
$$

If $u_{1}(z), u_{2}(z), w_{1}(z)$ and $w_{2}(z)$ are defined by the boundary conditions:

$$
\begin{aligned}
& u_{i}{ }^{(i-1)}(\zeta)=1, \quad w_{i}{ }^{(i-1)}(\zeta)=1, \quad(i, k=1,2 ; \\
& \left.u_{i}{ }^{(k-1)}(\zeta)=0, \quad w_{i}{ }^{(k-1)}(\zeta)=0, \quad k \neq i\right)
\end{aligned}
$$

then $v(z)$, any solution of (3), may be written in the form

$$
v(z)=v(\zeta) u_{1}(z) w_{1}(z)
$$




$$
\begin{aligned}
&-\frac{1}{4 r}\left[v^{\prime \prime \prime}+\right.\left.\left(6 q_{2}-2 r\right) v^{\prime}+3 q_{2}^{\prime} v\right]_{z=\zeta} u_{1}(z) w_{2}(z) \\
&+\frac{1}{4 r}\left[v^{\prime \prime \prime}+\left(6 q_{2}+2 r\right) v^{\prime}+3 q_{2}^{\prime} v\right]_{z=\zeta} u_{2}(z) w_{1}(z) \\
&+\frac{1}{2}\left[v^{\prime \prime}(\zeta)+3 q_{2}(\zeta) v(\zeta)\right] u_{2}(z) w_{2}(z) .
\end{aligned}
$$

$S(v, v)=0$ is therefore the necessary and sufficient condition that $v(z)$ be factorable as the product of a solution of (5) by a solution of (6). Therefore, if $S(v, v)=0$ the number of zeros of $v(z)$ in a given closed interval $(a, b)$ will be limited by Sturm's theorems concerning the zeros of solutions of (5) and (6). If, for example, the equations (5) and (6) have $k_{1}$ and $k_{2}$ for their respective indices of oscillation in $(a, b)$ then every solution of (5) [(6)] will have $k_{1}$ or $k_{1}+1$ [ $k_{2}$ or $\left.k_{2}+1\right]$ zeros in the interval $(a, b)$, and every solution, $v(z)$, of (3) for which $S(v, v)=0$ will have $k_{1}+k_{2}, k_{1}+k_{2}+1$, or $k_{1}+k_{2}+2$ zeros in $(a, b)$. If, in particular, $q_{2}(z)<0$ and $0<C<9 q_{2}{ }^{2}(z)$ throughout $(a, b)$ then the coefficients of $u(z)$ and $w(z)$ in (5) and (6) will be negative throughout $(a, b)$ and $k_{1}=k_{2}=0 .^{*}$ Therefore, if $q_{2}(z)<0$ and $0<C<9 q_{2}{ }^{2}(z)$ throughout $(a, b)$ then no solution of (3) for which $S(v, v)=0$ has more than two zeros in $(a, b)$, and some such solutions have no zeros in $(a, b)$.

WESLEYAN UNIVERSITY, Middletown, ConN.

\section{AN ACKNOWLEDGMENT OF PRIORITY.}

BY PROFESSOR A. A. BENNETT.

IN the July, 1919, number of the Bulletin (volume 25, page 455), I gave a solution condensed to about two pages, of a problem proposed by the Philosophical Faculty of the University of Berlin as a prize problem. My attention has been called to the fact that this question is completely handled in an analogous manner by Harold Hilton, in his book on "Homogeneous Linear Substitutions," 1914, in Chapter VI, section 8. Similar results are given in the same chapter for

* See Bôcher's Méthodes de Sturm, Paris, 1917, p. 52. 